\title{
Medical Device-Associated Candida Infections in a Rural Tertiary Care Teaching Hospital of India
}

\author{
Sachin C. Deorukhkar and Santosh Saini \\ Department of Microbiology, Rural Medical College, Pravara Institute of Medical Sciences (Deemed University), \\ Loni, Maharashtra 413736, India \\ Correspondence should be addressed to Sachin C. Deorukhkar; deorukhkar.sachin@gmail.com
}

Received 29 October 2015; Revised 30 December 2015; Accepted 10 January 2016

Academic Editor: Albert Eid

Copyright (C) 2016 S. C. Deorukhkar and S. Saini. This is an open access article distributed under the Creative Commons Attribution License, which permits unrestricted use, distribution, and reproduction in any medium, provided the original work is properly cited.

\begin{abstract}
Health care associated infections (HCAIs) add incrementally to the morbidity, mortality, and cost expected of the patient's underlying diseases alone. Approximately, about half all cases of HCAIs are associated with medical devices. As Candida medical device-associated infection is highly drug resistant and can lead to serious life-threatening complications, there is a need of continuous surveillance of these infections to initiate preventive and corrective measures. The present study was conducted at a rural tertiary care hospital of India with an aim to evaluate the rate of medical device-associated Candida infections. Three commonly encountered medical device-associated infections (MDAI), catheter-associated urinary tract infection (CA-UTI), intravascular catheter-related blood stream infections (CR-BSI), and ventilator-associated pneumonia (VAP), were targeted. The overall rate of MDAI in our hospital was 2.1 per 1000 device days. The rate of Candida related CA-UTI and CR-BSI was noted as 1.0 and 0.3 , respectively. Untiring efforts taken by team members of Hospital Acquired Infection Control Committee along with maintenance of meticulous hygiene of the hospital and wards may explain the low MDAI rates in our institute. The present surveillance helped us for systematic generation of institutional data regarding MDAI with special reference to role of Candida spp.
\end{abstract}

\section{Introduction}

Health care associated infections (HCAIs) or hospital acquired infections (HAIs) are infections that occur during hospitalization but are neither present nor incubating upon hospital admission. Various factors like increasing incidence of hospitalization, rapid advancement in medical technology, and injudicious use of antibiotics along with better adaptation of microbes to the hospital environment contribute to exponential increase in HCAIs [1]. Even in developed nations, HCAIs concern $5-15 \%$ of hospitalized patients and can lead to complications in $25-50 \%$ of those admitted in intensive care units (ICUs) [2].

HCAIs add incrementally to the morbidity, mortality, and cost expected of the patient's underlying diseases alone. Additionally, these infections have the potential to severely undermine the superlative effect of the clinician. Therefore, prevention and control of HCAIs are an ever escalating threat which needs to be expeditiously managed.

Approximately about half all cases of HCAIs are associated with medical devices [3]. In recent years, mycotic pathogens are increasingly reported as causes of HCAIs. Fungi, especially Candida spp., rank 3rd among various leading cause of catheter-associated infections [4]. Candida spp. can colonize and form biofilm on most, if not all, medical devices in current use [5]. As Candida medical deviceassociated infection is highly drug resistant and can lead to serious life-threatening complications, there is a need of continuous surveillance of these infections to initiate preventive and corrective measures. A number of published literatures on rate of medical device-associated infections (MDAI) are focused on bacterial organisms but unfortunately till date there is a paucity of data on Candida MDAI. Therefore, the present study was conducted at a rural tertiary care hospital 
of India with an aim to evaluate the rate of medical deviceassociated Candida infections.

\section{Materials and Methods}

The study was conducted in the Department of Microbiology, Rural Medical College and Hospital of Pravara Institute of Medical Sciences (Deemed University), Loni, Maharashtra, India. The protocol of the study was approved by Institutional Ethics Committee.

In this study, the following three commonly encountered medical device-associated infections (MDAI), catheterassociated urinary tract infection (CA-UTI), intravascular catheter-related blood stream infections (CR-BSI), and ventilator-associated pneumonia (VAP), as per the definition of CDC's National Nosocomial Infections Surveillance (NNIS) system criteria, were targeted.

The study was conducted for a period of two years (January 2013 to December 2014) and included patients who were admitted to ICU and different wards for more than $48 \mathrm{~h}$ and were exposed to medical devices like urinary catheters, intravascular catheter, and endotracheal tubes.

Laboratory methods employed for diagnosis of CA-UTI, CR-BSI, and VAP were as follows.

2.1. CA-UTI. In case of CA-UTI, urine sample was collected aseptically from sampling port of indwelling urinary catheter with sterile syringe and needle. The urine sample was inoculated on blood agar, MacConkey's agar, and Sabouraud dextrose agar (SDA) and incubated at $35^{\circ} \mathrm{C}$ for $24-48 \mathrm{~h}$.

The patient was labeled as a case of CA-UTI, when he/she developed one or more of the following conditions: temperature $\left(\geq 38^{\circ} \mathrm{C}\right)$, urgency, suprapubic tenderness, presence of yeast cells in Gram stained smear prepared from centrifuged urine sample, and isolation of yeast from urine as a pure growth with colony count $>10^{4}$ colony forming units (CFUs)/mL $[6,7]$. In case of bacteriuria, colony count $>10^{5} \mathrm{CFU} / \mathrm{mL}$ was considered significant.

2.2. CR-BSI. The CR-BSI was suspected when a patient with central venous catheter developed fever or other symptoms of sepsis of unknown origin. In such cases, two blood samples were aseptically collected; the first blood sample was obtained from the catheter itself and the second from the other arm. Specimens for blood culture were collected at the time of removal of central venous catheter. The central venous catheter was removed by the treating physician following all aseptic precautions and the distal $2 \mathrm{~cm}$ segment of the catheter was collected in sterile capped container with the help of sterile scissor. Inoculated blood culture bottles and catheter tip were immediately transported to microbiology laboratory.

The blood culture samples were processed as per standard microbiological protocols and catheter was processed as per semiquantitative method described by Maki et al. [8]. A colony count $>15 \mathrm{CFU} /$ plate was considered significant [8]. Isolation of the same organism from catheter and blood culture indicated CR-BSI.
2.3. VAP. VAP was suspected in a patient on mechanical ventilator when there was a development of new fever, cough, and purulent expectoration, supported by radiological evidence of a new or progressive pulmonary infiltrate and leukocytosis [9]. Bronchoalveolar lavage (BAL) and tracheal aspirate collected from these patients were inoculated on blood agar, MacConkey's agar, and SDA. The plates were incubated at $35^{\circ} \mathrm{C}$ for $24-48 \mathrm{~h}$. A colony count $>10^{3} \mathrm{CFU} / \mathrm{mL}$ (for $\mathrm{BAL}$ ) and $>10^{5} \mathrm{CFU} / \mathrm{mL}$ (for tracheal aspirate) were considered as significant [9].

Candida isolates were identified up to species level by assessing the formation of germ tubes, sugar assimilation, and colony color on HiChrome Candida agar (Himedia Laboratories Pvt. Ltd., Mumbai, India). HiCandida identification kit (Himedia Laboratories Pvt. Ltd., Mumbai, India) supplemented the species identification. Bacterial isolates were identified as per standard microbiological profile.

MDAI rate was expressed as the number of MDAI per 1000 device days and was calculated using the following formula [9]:

$$
\left(\frac{\text { Number of patients developing MDAI }}{\text { Total number of device days }}\right) \times 1000 \text {. }
$$

\section{Results}

During the study period, a total of 13456 patients were exposed to different types of medical devices for a total duration of 43621 days. Out of these, 93 (0.6\%) developed different types of MDAI. Bacterial infection was noted in $57(0.4 \%)$ patients, whereas Candida spp. were isolated from $36(0.2 \%)$ cases. The overall rate of MDAI in our hospital was 2.1 per 1000 device days. The overall rates of bacterial and Candida MDAI (1000 device days) were 1.3 and 0.8 , respectively (Table 1 ).

During the study period, out of 10621 patients with indwelling urinary catheters, only $80(0.7 \%)$ developed CAUTI. Bacterial CA-UTI was noted in $47(0.4 \%)$ cases, whereas Candida spp. were isolated from $33(0.3 \%)$ suspected cases of CA-UTI. The rate of catheter-associated urinary tract candidiasis per 1000 device days was 1.0 (Table 1).

As shown in Table 1, catheter-related blood stream Candida infection was noted in only $0.1 \%$ of patients exposed to central venous catheterization. The rate of catheter-related blood stream Candida infection per 1000 device days was 0.3 . In our study, no case of VAP due to Candida spp. was seen.

The spectrum of microorganisms isolated from various types of MDAI is shown in Table 2. Among bacterial pathogens, E. coli followed by Pseudomonas aeruginosa and Staphylococcus aureus were the predominant pathogens. C. albicans was isolated from 13 cases, whereas 23 isolates were non-C. albicans (NCA) spp. Among Candida isolates, predominance of $C$. tropicalis was noted.

As shown in Table 2, E. coli followed by P. aeruginosa and Enterococcus spp. were the major bacterial isolates from CAUTI. C. tropicalis was one of the most common Candida spp. isolated from CA-UTI (Table 2). A total of 03 (0.1\%) Candida spp. were isolated from CR-BSI. These included a single 
TABLE 1: Rate of medical device-associated infections.

\begin{tabular}{|c|c|c|c|c|c|c|c|}
\hline \multirow{2}{*}{$\begin{array}{l}\text { Type of MDAI } \\
\text { (number of cases) }\end{array}$} & \multirow{2}{*}{$\begin{array}{l}\text { Total number } \\
\text { of device days }\end{array}$} & \multicolumn{3}{|c|}{ Number of MDAI detected } & \multicolumn{3}{|c|}{ Infection rate (per 1000 device days) } \\
\hline & & Bacterial (\%) & Candida (\%) & Total (\%) & Bacterial & Candida & Total \\
\hline CA-UTI (10621) & 32310 & $47(0.4)$ & $33(0.3)$ & $80(0.7)$ & 1.4 & 1.0 & 2.4 \\
\hline CR-BSI (2632) & 10113 & $06(0.2)$ & $03(0.1)$ & $09(0.3)$ & 0.5 & 0.3 & 0.8 \\
\hline VAP (203) & 1198 & $04(1.9)$ & - & $04(1.9)$ & 3.3 & - & 3.3 \\
\hline Total (13456) & 43621 & $57(0.4)$ & $36(0.2)$ & $93(0.6)$ & 1.3 & 0.8 & 2.1 \\
\hline
\end{tabular}

TABLE 2: Spectrum of microorganism isolated from medical device associated infections.

\begin{tabular}{|c|c|c|c|c|}
\hline Organism & CA-UTI & CR-BSI & VAP & Total \\
\hline \multicolumn{5}{|c|}{ Bacterial isolates $(n=57)$} \\
\hline E. coli & 18 & 01 & 01 & 20 \\
\hline Klebsiella pneumoniae & 06 & 02 & 01 & 09 \\
\hline Pseudomonas aeruginosa & 09 & 01 & 01 & 11 \\
\hline Staphylococcus aureus & 02 & 01 & 01 & 04 \\
\hline Coagulase negative Staphylococci & 03 & 01 & - & 04 \\
\hline Enterococcus spp. & 09 & - & - & 09 \\
\hline Total & 47 & 06 & 04 & 57 \\
\hline \multicolumn{5}{|c|}{ Candida spp. $(n=36)$} \\
\hline C. albicans & 12 & 01 & - & 13 \\
\hline C. tropicalis & 14 & 01 & - & 15 \\
\hline C. glabrata & 05 & - & - & 05 \\
\hline C. krusei & 02 & - & - & 02 \\
\hline C. parapsilosis & - & 01 & - & 01 \\
\hline Total & 33 & 03 & - & 36 \\
\hline
\end{tabular}

isolate each of C. albicans, C. tropicalis, and C. parapsilosis (Table 2).

\section{Discussion}

HCAI is one of the most common adverse, iatrogenic events experienced by hospitalized patients. These infections have occurred since care of patients in hospitals began and health care providers have often been haunted by the maxim: "the patient initially responded to the treatment but later he died of an infection." In recent years, increasing usage of indwelling medical devices has led to an increase in HCAIs.

In the present study, the overall MDAI rate was found to be 2.1 per 1000 device days, which was higher than the rate reported by Singh et al. [9]. However, the rate of MDAI in our hospital was quite low than that reported in studies of Vonberg et al. [10] and Habibi et al. [11]. The rates of MDAI vary with the category of the hospital and health care unit studied. Untiring efforts taken by team members of Hospital Acquired Infection Control Committee (HAICC) along with maintenance of meticulous hygiene of the hospital and wards may explain the low MDAI rates in our institute. In addition, regular sensitization workshops on hospital infection control, hand hygiene, and universal standard precautions are conducted for health care providers of all cadres.
In the present surveillance, the rate of catheter-associated urinary tract candidiasis per 1000 device days was 1.0. Candiduria is an increasingly prevalent HCAI and is rarely noted as a community acquired infection in a healthy person with structurally normal urinary tract [6]. Approximately about $10-15 \%$ of health care associated UTIs are due to Candida spp. [7]. Presence of indwelling catheter is one of the important risk factors associated with candiduria [6]. Platt et al. reported Candida as the causative agent in $27 \%$ of all UTI related to indwelling catheters [12]. Candiduria in the presence of other risk factors can predispose to disseminated infections [7].

The predominance of NCA spp. over C. albicans was noted in our study. As per Fischer, the microbiology of candiduria is changing with $>50 \%$ of isolates now belonging to NCA spp. [13]. NCA spp. are not only better adapted to the urinary tract but also more difficult to eradicate than $C$. albicans [1]. The isolates from NCA spp. often demonstrate reduced susceptibility to commonly used antifungal drugs [1]. Therefore, species identification along with antifungal susceptibility testing aids in proper management of Candida infections.

C. tropicalis was the most common Candida spp. isolated from CA-UTI. This observation is in accordance with that of Jain et al. [7] where C. tropicalis was the most frequent cause of candiduria in catheterized ICU patients. In recent years, C. tropicalis alone, or in association with other species, is more frequently implicated in human infections $[14,15]$. As compared to C. albicans, C. tropicalis demonstrate reduced susceptibility to fluconazole [14].

In our study, the rate of bacterial CA-UTI 1000 device days was 1.4 with $E$. coli as the major bacterial pathogen. $E$. coli is the most common facultative anaerobic commensal of the gastrointestinal tract, which is in close proximity to the genitourinary tract. In addition, some strains bear surface fimbria notably type I fimbriae, which bind to latex catheters and urothelial cells [16]. Singh et al. reported only one case of CA-UTI caused by Enterococci spp. in MICU patient [9]. The rate of CA-UTI in their study was 0.6 per 1000 device days. Habibi et al. reported the CA-UTI rate as 11.3 per 1000 device days [11]. Various other studies have reported the rate of CAUTI ranging from 1.7 to 30 per 1000 device days [9].

Nosocomial bloodstream infections are one of the important causes of morbidity and mortality in hospitalized patients. In USA, more than 200,000 nosocomial bloodstream infections occur each year [17]. Rates of CR-BSI vary depending on the types of ICU, ranging from 2.9 to 11.3 per 1000 catheter days $[18,19]$. In a study conducted in 12 ICUs of 7 Indian hospitals, the CR-BSI rate was 7.92 per 1000 catheter 
days and excess mortality was 4\% [20]. In the present study, the rate of CR-BSI was noted as 0.8 per 1000 catheter days which is slightly higher than that reported by Singh et al. [9].

Candida spp. are the commonest cause of disseminated mycoses with reported mortality to be as high as 40-50\% [21]. They account for about $15 \%$ of total HCAIs and more than $72 \%$ of nosocomial mycoses [21]. Candida spp. are the fourth leading cause of BSI in USA but comparatively less common in Europe [22]. However, the exact prevalence of nosocomial Candida BSI in India is not known [23]. In our study, the rate of catheter-related blood stream Candida infection per 1000 device days was 0.3 . A total of $03(0.1 \%)$ Candida spp. were isolated from CR-BSI. These included one isolate each of $C$. albicans, C. tropicalis, and C. parapsilosis. C. parapsilosis has been described as the second or third most common Candida spp. responsible for BSI in Europe, Canada, Asia, and Latin America [24]. The capability of C. parapsilosis to form a biofilm on intravascular and prosthetic devices, selective growth in hyperalimentation solution, and colonization of human hand favor its survival and dissemination in hospital environment [25]. Long term use of central venous catheter or indwelling devices is an important risk factor for $C$. parapsilosis infection [25]. C. tropicalis is the most common cause of nosocomial Candida BSI in India [15]. This NCA $\mathrm{sp}$. has ability to develop rapid resistance to fluconazole. It is mostly isolated from ICU patients with prolonged indwelling catheters, receiving broad-spectrum antibiotic therapy or with malignancy [25]. C. tropicalis is also associated with high mortality rates compared to other NCA spp. and C. albicans [25]. Mansur et al. [26] and Kaur et al. [27] also reported significant isolation of NCA spp. from CR-BSI in their studies.

Klebsiella pneumoniae, E. coli, S. aureus, coagulase negative Staphylococci, and $P$. aeruginosa were the bacterial pathogens isolated from CR-BSI. Though the type of nosocomial pathogen varies as per the health care setup, the pathogens commonly associated with HCAI are microorganisms prevalent in the hospital environment [9].

VAP remains an important cause of morbidity and mortality despite advances in antimicrobial therapy, better supportive care modalities, and the use of a wide range of preventive measures [28]. It complicates the course of $8-28 \%$ of the patients on mechanical ventilation [28]. In the present study, the rate of VAP per 1000 device days was found to be 3.3 , which is quite low in comparison to that reported by other researchers.

K. pneumoniae, E. coli, S. aureus, and P. aeruginosa were the bacterial pathogens isolated from VAP cases. In the present study, no case of VAP due to Candida spp. was seen. This observation was in agreement with other researchers' like Singh et al. (2010) [9] and Lalwani et al. (2014) [28]. Therefore, it can be stated that VAP may be caused by a wide spectrum of bacterial pathogens but rarely due to mycotic pathogens.

\section{Conclusion}

Although all health care setups have infection control policies and staff takes every precaution to avoid infection, the risk of
HAI can never be completely eliminated. Therefore, surveillance of HAI is very important to understand the magnitude of the problem and to initiate intensified preventive measures for improvement in patient care. The present surveillance helped us for systematic generation of institutional data regarding three most important types of MDAI (CR-BSI, CAUTI, and VAP) with special reference to role of Candida spp. To the best of our knowledge, the present study is the first to report the scenario of medical device-associated Candida infections from rural part of India.

\section{Conflict of Interests}

The authors declare that there is no conflict of interests regarding the publication of this paper.

\section{Acknowledgments}

The authors would like to thank the management of Rural Medical College and Rural Hospital of Pravara Institute of Medical Sciences (Deemed University), Loni, Maharashtra, for their encouragement and support throughout the study. The authors acknowledge Mrs. Ujjwala Bhingardive, Infection Control Nurse, Pravara Rural Hospital, Loni, for her help in data collection.

\section{References}

[1] S. C. Deorukhkar, S. Saini, and S. Mathew, "Non-albicans Candida infection: an emerging threat," Interdisciplinary Perspectives on Infectious Diseases, vol. 2014, Article ID 615958, 7 pages, 2014.

[2] P. Eggimann and D. Pittet, "Infection control in the ICU," Chest, vol. 120, no. 6, pp. 2059-2093, 2001.

[3] E. M. Kojic and R. O. Darouiche, "Candida infections of medical devices," Clinical Microbiology Reviews, vol. 17, no. 2, pp. 255267, 2004.

[4] S. R. Lockhart, "Current epidemiology of Candida infection," Clinical Microbiology Newsletter, vol. 36, no. 17, pp. 131-136, 2014.

[5] C. J. Seneviratne, L. Jin, and L. P. Samaranayake, "Biofilm lifestyle of Candida: a mini review," Oral Diseases, vol. 14, no. 7, pp. 582-590, 2008.

[6] Z. A. Bukhary, "Candiduria: a review of clinical significance and management," Saudi Journal of Kidney Diseases and Transplantation, vol. 19, no. 3, pp. 350-360, 2008.

[7] M. Jain, V. Dogra, B. Mishra, A. Thakur, S. L. Loomba, and A. Bhargava, "Candiduria in catheterized intensive care unit patients: emerging microbiological trends," Indian Journal of Pathology and Microbiology, vol. 54, no. 3, pp. 552-555, 2011.

[8] D. G. Maki, C. E. Weise, and H. W. Sarafin, "A semiquantitative culture method for identifying intravenous-catheter-related infection," The New England Journal of Medicine, vol. 296, no. 23, pp. 1305-1309, 1977.

[9] S. Singh, Y. Pandya, R. Patel, M. Paliwal, A. Wilson, and S. Trivedi, "Surveillance of device-associated infections at a teaching hospital in rural Gujarat-India," Indian Journal of Medical Microbiology, vol. 28, no. 4, pp. 342-347, 2010.

[10] R.-P. Vonberg, M. Behnke, C. Geffers et al., "Device-associated infection rates for non-intensive care unit patients," Infection 
Control and Hospital Epidemiology, vol. 27, no. 4, pp. 357-361, 2006.

[11] S. Habibi, N. Wig, S. Agarwal et al., "Epidemiology of nosocomial infections in medicine intensive care unit at a tertiary care hospital in northern India," Tropical Doctor, vol. 38, no. 4, pp. 233-235, 2008.

[12] R. Platt, B. F. Polk, B. Murdock, and B. Rosner, "Risk factors for nosocomial urinary tract infection," American Journal of Epidemiology, vol. 124, no. 6, pp. 977-985, 1986.

[13] J. F. Fisher, "Candida urinary tract infections-epidemiology, pathogenesis, diagnosis, and treatment: executive summary," Clinical Infectious Diseases, vol. 52, supplement 6, pp. S429S432, 2011.

[14] R. J. Kothavade, M. M. Kura, A. G. Valand, and M. H. Panthaki, "Candida tropicalis: its prevalence, pathogenicity and increasing resistance to fluconazole," Journal of Medical Microbiology, vol. 59, no. 8, pp. 873-880, 2010.

[15] S. C. Deorukhkar, S. Saini, and S. Mathew, "Virulence factors contributing to pathogenicity of Candida tropicalis and its antifungal susceptibility profile," International Journal of Microbiology, vol. 2014, Article ID 456878, 6 pages, 2014.

[16] K. J. Dowling, J. A. Roberts, and M. B. Kaack, "P-fimbriated Escherichia coli urinary tract infection: a clinical correlation," Southern Medical Journal, vol. 80, no. 12, pp. 1533-1536, 1987.

[17] National Nosocomial Infections Surveillance System, "National Nosocomial Infections Surveillance (NNIS) System report, data summary from January 1992 through June 2004, issued October 2004," American Journal of Infection Control, vol. 32, no. 8, pp. 470-485, 2004.

[18] V. D. Rosenthal, D. G. Maki, R. Salomao et al., "Deviceassociated nosocomial infections in 55 intensive care units of 8 developing countries," Annals of Internal Medicine, vol. 145, no. 8, pp. 582-591, 2006.

[19] C. A. Moreno, V. D. Rosenthal, N. Olarte et al., "Deviceassociated infection rate and mortality in intensive care units of 9 Colombian hospitals: findings of the International Nosocomial Infection Control Consortium," Infection Control and Hospital Epidemiology, vol. 27, no. 4, pp. 349-356, 2006.

[20] A. Mehta, V. D. Rosenthal, Y. Mehta et al., "Device-associated nosocomial infection rates in intensive care units of seven Indian cities. Findings of the International Nosocomial Infection Control Consortium (INICC)," Journal of Hospital Infection, vol. 67, no. 2, pp. 168-174, 2007.

[21] L.-Y. Chen, S.-Y. Liao, S.-C. Kuo et al., "Changes in the incidence of candidaemia during 2000-2008 in a tertiary medical centre in northern Taiwan," Journal of Hospital Infection, vol. 78, no. 1, pp. 50-53, 2011.

[22] M. Méan, O. Marchetti, and T. Calandra, "Bench-to-bedside review: Candida infections in the intensive care unit," Critical Care, vol. 12, no. 1, article 204, 2008.

[23] A. Chakrabarti, K. Singh, and S. Das, "Changing face of nosocomial candidemia," Indian Journal of Medical Microbiology, vol. 17, no. 4, pp. 160-166, 1999.

[24] S. T. Tay, S. L. Na, and J. Chong, "Molecular differentiation and antifungal susceptibilities of Candida parapsilosis isolated from patients with bloodstream infections," Journal of Medical Microbiology, vol. 58, no. 2, pp. 185-191, 2009.

[25] S. Silva, M. Negri, M. Henriques, R. Oliveira, D. W. Williams, and J. Azeredo, "Candida glabrata, Candida parapsilosis and Candida tropicalis: biology, epidemiology, pathogenicity and antifungal resistance," FEMS Microbiology Reviews, vol. 36, no. 2, pp. 288-305, 2012.
[26] F. J. Mansur, L. Barai, M. M. Karim, J. A. Haq, K. Fatema, and M. O. Faruq, "Intravascular catheter related infections and antimicrobial susceptibility pattern of isolated bacteria in a tertiary care hospital of Bangladesh," Indian Journal of Medical Microbiology, vol. 32, no. 1, pp. 68-71, 2014.

[27] M. Kaur, V. Gupta, S. Gombar, J. Chander, and T. Sahoo, "Incidence, risk factors, microbiology of venous catheter associated bloodstream infections-a prospective study from a tertiary care hospital," Indian Journal of Medical Microbiology, vol. 33, no. 2, pp. 248-254, 2015.

[28] S. Lalwani, P. Mathur, V. Tak et al., "Diagnosis of ventilatorassociated pneumonia: comparison between ante-mortem and post-mortem cultures in trauma patients," Indian Journal of Medical Microbiology, vol. 32, no. 3, pp. 294-300, 2014. 


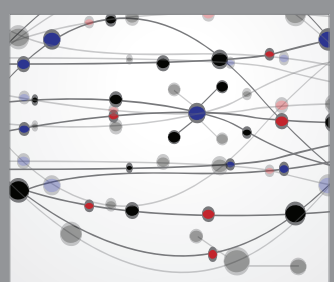

The Scientific World Journal
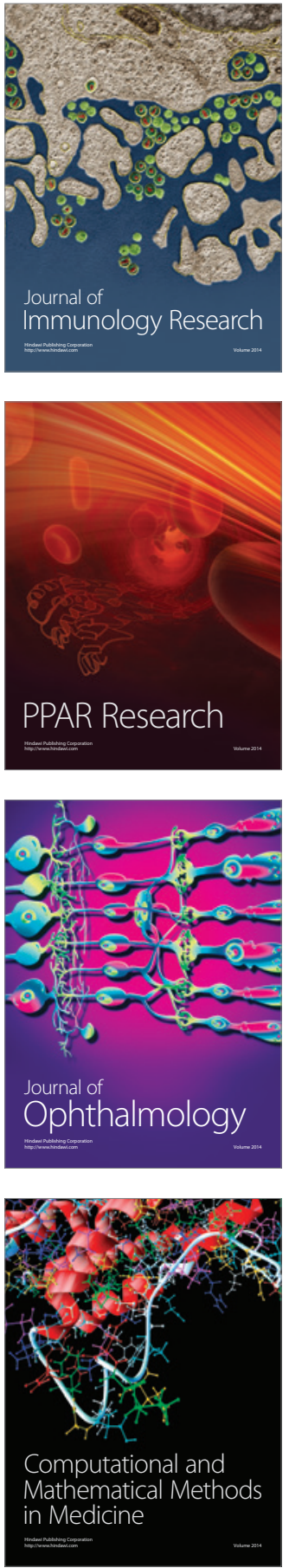

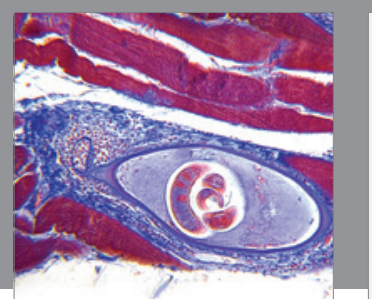

Gastroenterology Research and Practice

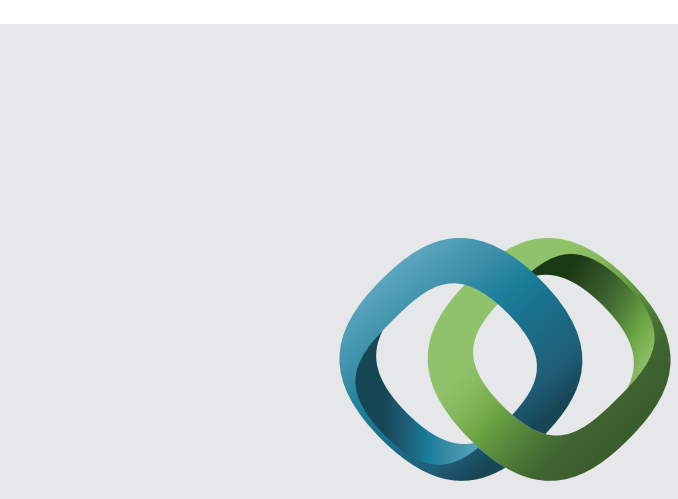

\section{Hindawi}

Submit your manuscripts at

http://www.hindawi.com
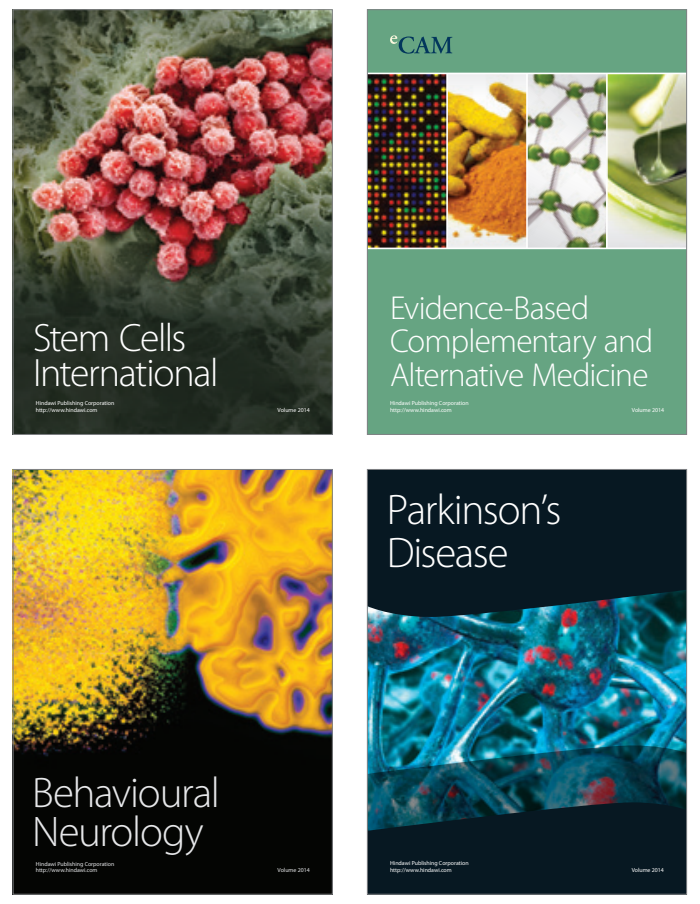
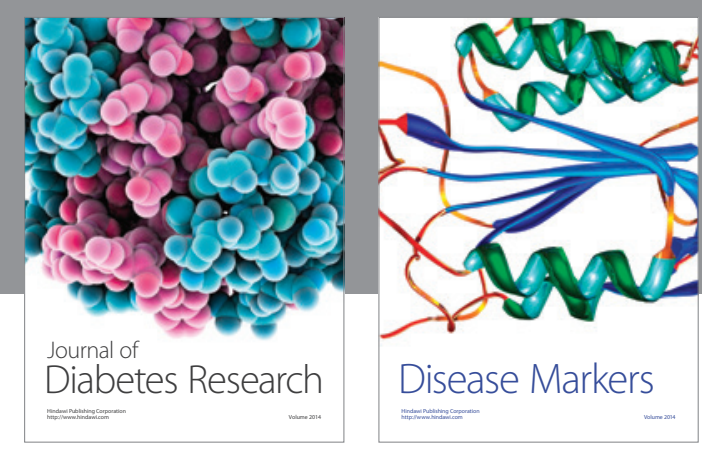

Disease Markers
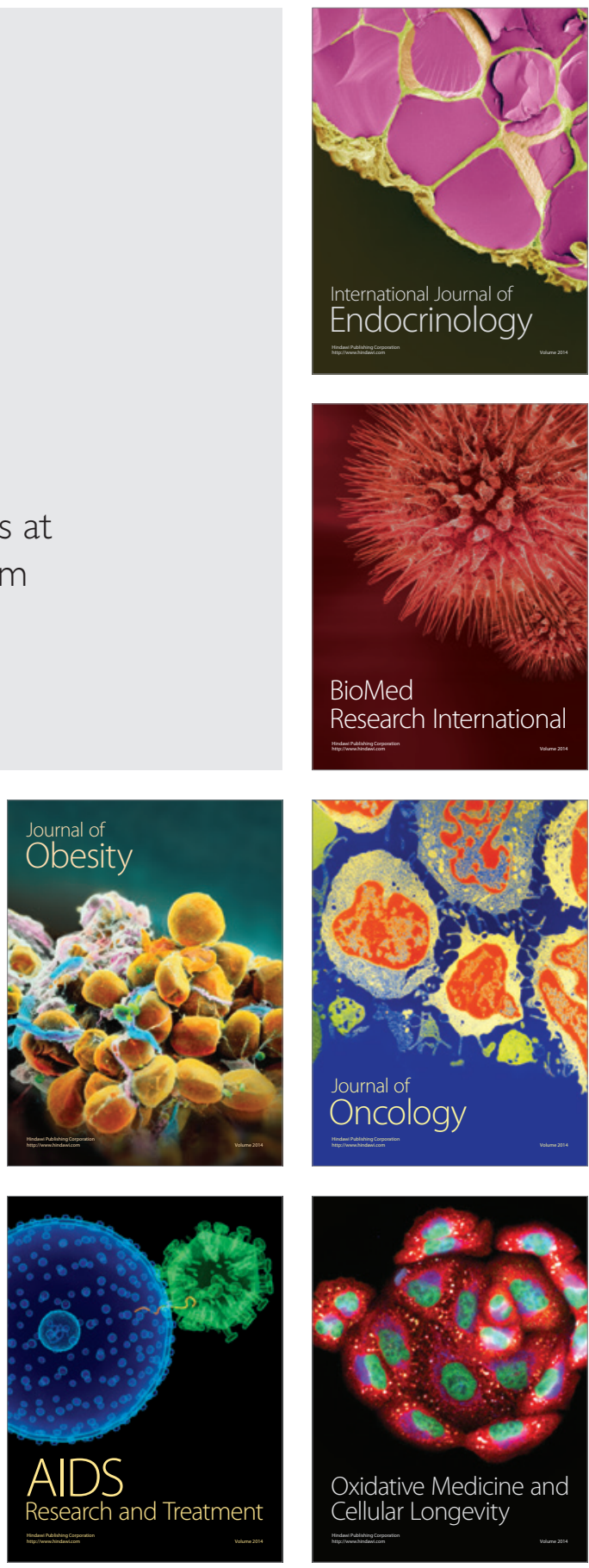